\title{
Complex Events in the Evolution of the Human Pseudoautosomal Region 2 (PAR2)
}

\author{
Fadi J. Charchar, ${ }^{1,6,8}$ Marta Svartman, ${ }^{1,2,7}$ Nisrine El-Mogharbel, ${ }^{1,3,7}$ \\ Mario Ventura, ${ }^{4}$ Patrick Kirby, ${ }^{1,3}$ Maria R. Matarazzo, ${ }^{5}$ Alfredo Ciccodicola, ${ }^{5}$ \\ Mariano Rocchi, ${ }^{4}$ Maurizio D'Esposito, ${ }^{5}$ and Jennifer A. Marshall Graves ${ }^{1,3}$ \\ ${ }^{1}$ Department of Genetics, La Trobe University, Bundoora, Victoria 3086, Australia; ${ }^{2}$ Departamento de Biologia, Instituto de \\ Biociências, Universidade de São Paulo, São Paulo-SP, Brasil; ${ }^{3}$ Comparative Genomics Group, Research School of Biological \\ Sciences, Australian National University, Canberra, ACT 2601, Australia; ${ }^{4}$ Dipartimento di Anatomia Patologica e di \\ Genetica, Sezione di Genetica, Bari, Italy; ${ }^{5}$ Institute of Genetics and Biophysics "A. Buzzati Traverso," Naples, Italy. \\ University of Glasgow, Department of Medicine and Therapeutics, Western Infirmary, G11 6NT, UK.
}

\begin{abstract}
The 320-kb human pseudoautosomal region 2 (PAR2) at the tips of the long arms of the $\mathrm{X}$ and $\mathrm{Y}$ chromosomes is thought to have been duplicated onto the $Y$ chromosome recently in primate evolution. The four genes within PAR2 have been proposed to constitute two zones with different base ratios and transcription, one of which was added recently to the $\mathrm{X}$ chromosome. To test this hypothesis, we cloned and mapped PAR2 genes in other species, the lemur, the cat, and a marsupial, the tammar wallaby. None of the human PAR2 genes colocalized with human PARI genes in the marsupial genome, confirming that the human PAR1 and PAR2 evolved independently. Of the four PAR2 genes, only SYBLI was located on the $X$ chromosome in all species, including marsupials, so it was part of the ancient $X$. HSPRY3 localized to the $\mathrm{X}$ in all the eutherians, but not marsupial, so it must have been added to the $X$ 80-130 million years ago. CXYorf1 was present on the $X$ in primates and also in mouse, but autosomal in wallaby, suggesting a later addition 70-130 million years ago, and IL9R was on the $\mathrm{X}$ only in primate, suggesting addition 60-70 million years ago. The results therefore demonstrate that at least two independent additions were necessary for PAR2 evolution. The present gene order on the human $\mathrm{X}$ also requires two inversions. The complicated evolutionary pathway supports the hypothesis that terminal interchromosomal rearrangements are common in regions unpaired at meiosis.
\end{abstract}

[The sequence data from this study have been submitted to GenBank under accession nos. AF544202, AF544203, AF544204, and AF544205.]

The human $\mathrm{X}$ and $\mathrm{Y}$ chromosomes differ in size, morphology, and gene content, the $\mathrm{X}$ being large and gene rich and the $\mathrm{Y}$ being small and heterochromatic. They do not pair at male meiosis except within small, homologous, "pseudoautosomal" regions (PARs) (Vogt et al. 1997). PARs lie at either extremity of the human sex chromosomes (Cooke et al. 1985). The 2.6-Mb PAR region 1 (PAR1), at the tip of the short arm (Xp-YpPAR) contains 13 genes (Rappold 1993; Gianfrancesco et al. 2001b), and is required for pairing of the $\mathrm{X}$ and $\mathrm{Y}$ chromosomes at male meiosis. The 320-kb PAR region 2 (PAR2) at the end of the long arms (Xq-YqPAR) (Freije et al. 1992) shows a much lower frequency of pairing and recombination than PAR1 and is not necessary for fertility (Kvaloy et al. 1994; Li and Hamer 1995; Kuhl et al. 2001).

The first two genes to be mapped to the PAR2 were SYBL1 (synaptobrevin-like protein 1 ) and IL9R (interleukin 9 receptor). Recently, the entire human PAR2 was sequenced and found to contain two other genes HSPRY3 (homolog to Drosophila sprouty 3 ) and CXYorf1, as well as a number of frag-

\footnotetext{
${ }^{7}$ These authors contributed equally to this work. ${ }^{8}$ Corresponding author. E-MAIL fjc4a@clinmed.gla.ac.uk; FAX +44 1412111763. Article and publication are at http://www.genome.org/cgi/doi/10.1101/ gr.390503. Article published online before print in January 2003.
}

mentary pseudogenes (Ciccodicola et al. 2000). The order of the genes from centromere to telomere is HSPRY3, SYBL1, IL9R, and CXYorf1. HSPRY3 and SYBL1 lie within the proximal $100 \mathrm{~kb}$, while IL9R and CXYorf1 are close together in the GC-rich distal $35 \mathrm{~kb}$. HSPRY3 and SYBL1 both map to the X, but not the $\mathrm{Y}$, in primate and the mouse. IL9R maps to the $\mathrm{X}$ in primate but is autosomal in mouse (Kermouni et al. 1995; D'Esposito et al. 1997; Vermeesch et al. 1997; Matarazzo et al. 1999; Ciccodicola et al. 2000) (Table 1). HSPRY3 and SYBL1 are both inactive on the $\mathrm{Y}$ and are subject to $\mathrm{X}$ inactivation in humans. In contrast, IL9R and CXYorf1 are expressed from the $\mathrm{Y}$ and are not subject to $\mathrm{X}$ inactivation (Huber et al. 1999; Ciccodicola et al. 2000).

The presence of these four genes on the $\mathrm{X}$ but not the $\mathrm{Y}$ in primate and mouse indicated that the region was transferred to the $\mathrm{Y}$ during the last few million years, perhaps via an illegitimate LINE sequence recombination between the $X$ and Y (Kvaloy et al. 1994). The absence of IL9R from the mouse $\mathrm{X}$ and the dichotomy in expression patterns between proximal and distal pairs of PAR2 genes led to the hypothesis that the regions containing them were added independently to the $\mathrm{X}$ chromosome during eutherian evolution. Ciccodicola et al. (2000) suggested a division into Zone 1 (HSPRY3 and SYBL1) and a later added Zone 2 (IL9R and CXYorf1) possibly obtained by three independent events. As a result, there 
Table 1. Map Position of PAR2 Genes in Different Mammalian Species

\begin{tabular}{|c|c|c|c|c|c|c|c|c|}
\hline & & \multicolumn{6}{|c|}{ Location in } & \multirow{2}{*}{$\begin{array}{c}\text { Time added to } \\
\text { X (MY) }\end{array}$} \\
\hline & & human & apes & lemur & mouse & cat & wallaby & \\
\hline \multirow[t]{2}{*}{ Divergence from human (MY) } & & & 15 & 60 & 70 & 80 & 130 & \\
\hline & HSPRY3 & $X / Y$ & $x$ & $x$ & $x$ & $x$ & 3 & $80-130$ \\
\hline \multirow[t]{3}{*}{ PAR2 Genes } & SYBL1 & $X / Y$ & $x$ & $x$ & $x+A$ & $?$ & $x$ & $>130$ \\
\hline & $I L 9 R$ & $X / Y+A$ & $x$ & $X+A$ & 11 & A & 1 & $60-70$ \\
\hline & CXYorfl & $X / Y+A$ & $X+A$ & & $x$ & & 3 & $70-130$ \\
\hline
\end{tabular}

MY indicates million years

A indicates localization on an autosome(s)

? indicates no positive identified.

are differences in base composition, recombination, and transcription that define operationally the two PAR2 zones.

We tested this hypothesis by comparing the location of homologs of PAR2 genes in two eutherian mammals that diverged from humans 60-70 million years ago (Mya) and in a distantly related marsupial mammal, which diverged independently from the eutherian lineage 130 Mya (Kumar and Hedges 1998). Comparative mapping of human X-borne genes in distantly related mammals can distinguish genes that were a part of the ancient mammalian X, and have been important in establishing the origin of the human PAR1. Mapping human X-borne genes in marsupial and monotreme mammals, which diverged from the eutherian lineage 130 and 170 Mya, respectively, have defined a conserved region (XCR) shared by the X chromosome in all three extant mammals, and a region (XAR) recently added to the eutherian $\mathrm{X}$, but still autosomal in marsupials and monotremes (Graves 1995; Graves et al. 1998). The eutherian Y is also composed of a conserved (YCR) and an added region (YAR) that contains most of the ubiquitously expressed genes (Waters et al. 2001). The demonstration that cloned marsupial homologs of human PAR1 genes colocalize with other genes on human Xp (Toder and Graves 1998) implied that PAR1 is part of the large region added to the eutherian $\mathrm{X}$ and $\mathrm{Y}$ after the divergence of marsupials (130 Mya) but before the eutherian radiation (80 Mya).

To examine the origin of PAR2, we therefore cloned and mapped all four human PAR2 genes in a model marsupial species, Macropus eugenii (the tammar wallaby). We also cloned and mapped two PAR2 genes in Felis cattus (the domestic cat) and Lemur catta (the lemur). If the human PAR2 region originated as part of the conserved region present on the $\mathrm{X}$ in all mammals, we would expect the human PAR2 genes to map to the $X$ also in marsupials. If PAR2 represents part of the same addition as PAR1, we would expect PAR2 genes to map with PAR1 genes on tammar 5p, and if PAR2 represents an independent addition, they will map on other autosomes. Our results further clarify PAR2 evolution, implying that most of PAR2 was independently added to the eutherian $X$ and rearranged in at least four separate events and before it was transposed to the $\mathrm{Y}$.

\section{RESULTS}

We cloned and characterized the wallaby homologs of all four PAR2 genes, the lemur and cat homologs of the human PAR2 genes, HSPRY3, SYBL1, and IL9R, and mapped their position in the tammar, lemur, and cat genomes.

\section{Isolation and Mapping of PAR2 Gene Homologs in Tammar Wallaby}

We screened a size-selected $M$. eugenii $\lambda$ genomic library of more than 360,000 recombinant phage with cDNA probes for human HSPRY3, SYBL1, IL9R, and CXYorf1. Clones were isolated for each gene. Hybridization of SalI/EcoRI-digested positive $\lambda$ clones with human HSPRY3, IL9R, and CXYorf1 cDNA yielded clear and distinct bands (data not shown) that were subcloned into plasmid vector and partially sequenced to confirm clone identity. The marsupial clones displayed $>80 \%$ homology to their human homologs within coding regions and were therefore confirmed as marsupial homologs of the human PAR2 genes. HSPRY3 clones were of two types, one of which was highly homologous to human HSPRY3, and the other was identical to the related SPRY1 gene, which has been previously cloned and mapped (Charchar et al. 2000). A single $S Y B L 1$ positive clone was sequenced after subcloning with TOPO Shotgun cloning kit (Invitrogen).

The tammar clones were each mapped to tammar chromosomes by fluorescence in situ hybridization (FISH). HSPRY 3 and CXYorf1 hybridized to the long arm of chromosome 3 in a medial position (Fig. 1A,B). Tammar IL9R hybridized to the tip of the long arm of chromosome 1 and SYBL1 mapped to the long arm of the X chromosome (Fig. 1D,E). FISH of HSPRY3 followed by hybridization with tammar wallaby chromosome 3 paint was used to check if HSPRY3 and CXYorf1 mapped to chromosome 3 or 4 , which are not distinguishable by size and morphology only (Fig. 1C).

Simultaneous hybridization of CXYorf1 and HSPRY 3 confirmed that these genes are located very close together at $3 \mathrm{q}$ (Fig. 1F-1H).

\section{Mapping of Human PAR2 Genes in Lemur and Cat}

We screened a size-selected male bacterial artificial chromosome (BAC) library from lemur and cat and isolated single clones for HSPRY3, SYBL1, and IL9R in the lemur. We also isolated single clones for HSPRY3 and $I L 9 R$ in the cat. There was no positive signal for $S Y B L I$ in the cat. This represents a homologous gene from each PAR2 zone. The cat and lemur clones were each mapped to cat and lemur chromosomes respectively by FISH.

IL9R hybridized to autosome E3 in the cat (Fig. 1I) and to the $\mathrm{X}$ chromosome and telomeres of an autosome of the lemur (Table 1). SYBL1 mapped to the long arm of the X chromosome in the lemur. HSPRY3 hybridized to the $\mathrm{X}$ chromosome in both cat (Table 1) and lemur (Fig. 1J). 


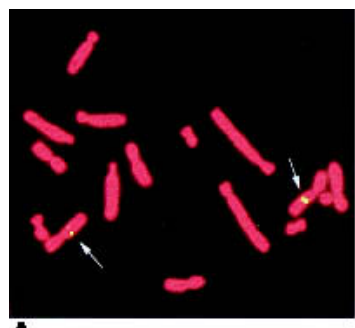

A

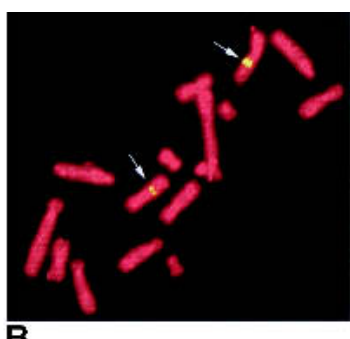

$\mathbf{B}$

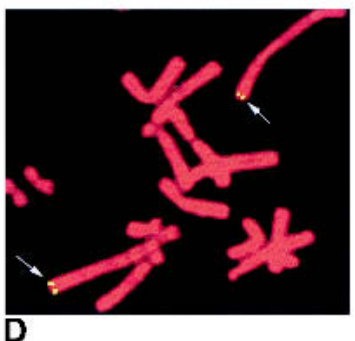

D

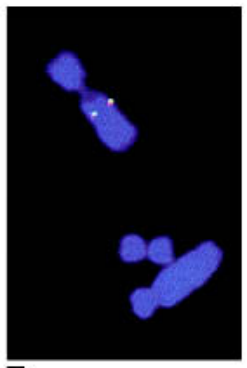

F

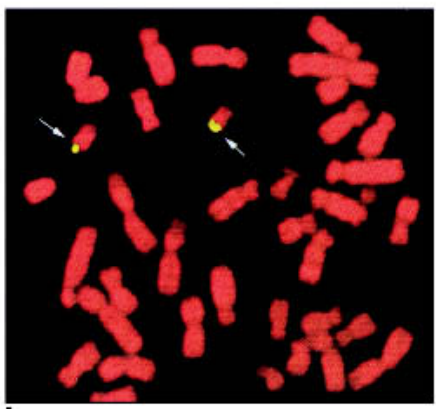

I

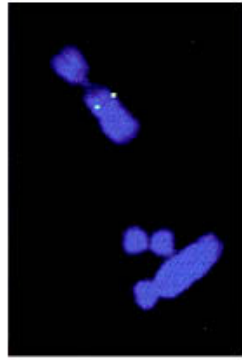

$\mathbf{G}$

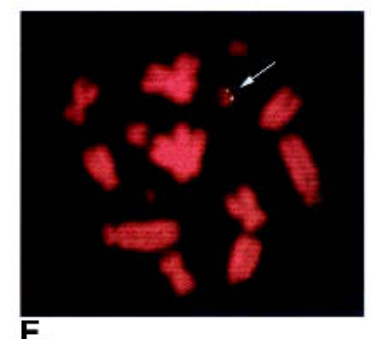

$\mathbf{E}$

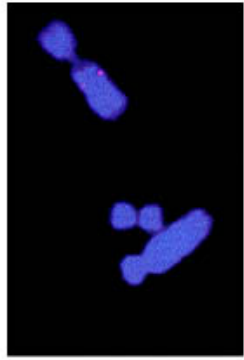

$\overline{\mathbf{H}}$

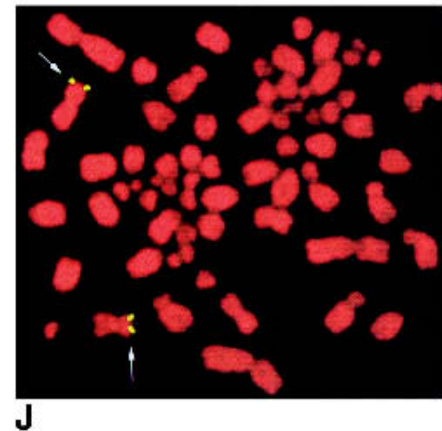

Figure 1 Chromosomal localization of the marsupial, cat and lemur orthologs as determined by fluorescence in situ hybridization analysis. (A) and (B) Localization of the HSPRY3 and CXYorf1 genes on chromosome 3. (C) The position is confirmed after sequential hybridization with HSPRY3 and tammar chromosome 3 paint. (D) and (E) IL9R and SYBL1 hybridized, respectively, to chromosome 1 and X. (F) Simultaneous hybridization of CXYorf1 and HSPRY3 to chromosome 3 where CXYorf1 is yellow (G) and HSPRY3 signal is red (H). (I) and (J) Chromosomal localization of the cat and lemur orthologs as determined by fluorescence in situ hybridization analysis. (I) Localization of IL9R to chromosome E3 in the cat, and (J) HSPRY 3 to the $\mathrm{X}$ chromosome in the Lemur.

\section{DISCUSSION}

\section{Evolution of PAR 2 Region-Are There Two Evolutionary Zones?}

The aims of this study were to test alternative theories for the origin of the human PAR2 region; either as part of the conserved region XCR present on the $\mathrm{X}$ in all mammals, or as part

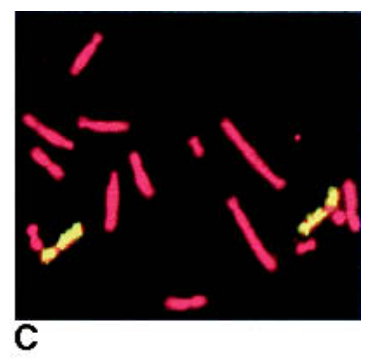

of an added region, either with PAR1, or as an independent addition. The first hypothesis requires that PAR2 genes lie on the $\mathrm{X}$ in other eutherians and marsupials as well as primates, and the second that they are autosomal in marsupials; on tammar chromosome $5 p$, if it was part of the same addition as PAR1, or elsewhere if it represents an independent addition. Our findings are hard to reconcile with any of these simple hypotheses.

The hypothesis that HSPRY3 and SYBL1 (zone 1) were part of the original eutherian $\mathrm{X}$, but $I L 9 R$ and CXYorf1 (zone 2) were transferred later would be supported by evidence that zone 1 genes map together on the $\mathrm{X}$ in all mammal species whereas zone 2 genes map together on the $X$ in species closely related to human but not on an autosome in distantly related species. HSPRY 3 and SYBL1 lie on the $X$ in all eutherian species, whereas $I L 9 R$ is on the $\mathrm{X}$ in all primates, but autosomal in mouse and cat. This suggests that HSPRY3 and SYBL1 were part of the original eutherian $\mathrm{X}$, but IL9R was added to the primate $X$ after the divergence of the cat and the rodent lineage 70 and 80 Mya.

However, CXYorf1, the other Zone 2 gene, is located on the mouse $X$, implying that it was added earlier than IL9R. Our results from the wallaby further complicate the picture, since $S Y B L 1$ was also on the $X$ chromosome, whereas the other Zone 1 gene HSPRY3 was autosomal. The two Zone 2 genes IL9R and CXYorf1 were both autosomal.

These results imply that $S Y B L 1$ was part of an original therian $\mathrm{X}$ in a common ancestor of eutherians and marsupials 130 Mya, and supports the results from cat and mouse. However, HSPRY3, being on the $\mathrm{X}$ in all eutherians but autosomal in wallaby, must have been added some time between the divergence of eutheria and marsupials 130 Mya and the eutherian radiation about 80 Mya. The two zone 2 genes $I L 9 R$ and CXYorf1 were also added to the $\mathrm{X}$ in independent events, since CXYorf1 was present on the $\mathrm{X}$ in mouse, whereas $I L 9 R$ was autosomal in mouse and cat and was therefore added only about 60-70 Mya. This independence is also favored by the different map positions of $I L 9 R$ and CXYorf1 in the wallaby, which suggests that the genes were added independently from different original sites. Thus 


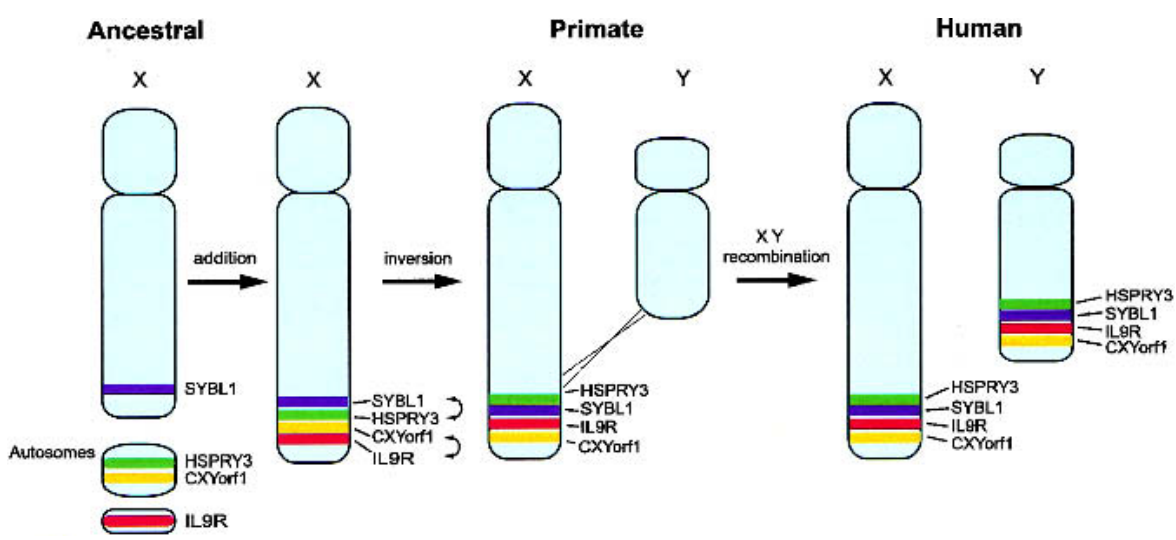

Figure 2 Schematic representation of the evolution of the PAR2 on the human $X$ chromosome. SYBL1 lies at a telomeric position on the original therian X. HSPRY3 and CXYorf1 were added first followed by an inversion to make HSPRY3 proximal to SYBL1. IL9R was added later, and then a second inversion occurred to make CXYorf1 telomeric.

the results do not support the reality of either Zone 1 or Zone 2. The subdivision of the PAR2 into two zones is based on the different molecular and transcriptional status, rather than their evolutionary history. The differences in base composition and transcription between the pairs of genes on the human $\mathrm{X}$ and $\mathrm{Y}$ are therefore more likely to represent subsequent adaptations, as for example their position with respect to $\mathrm{Y}$ heterochromatin.

\section{Independent Events in the Evolution of PAR2}

Our results are more compatible with the hypothesis that three of the four genes within the human PAR2 were added to the $\mathrm{X}$ chromosome in at least two separate steps. The additions could be dated by the separation times of the species in which they were $X$ linked or autosomal.

Our localization of SYBL1 on the X in the lemur and tammar is consistent with its $\mathrm{X}$ location in other primates and mouse, as well as an early report that it lies on the $\mathrm{X}$ in a related marsupial, the potoroo, Potorous tridactylus (Ciccodicola et al. 2000). However, signal was detected on the short arm of the compound potoroo $\mathrm{X}$, which is known from other studies to represent an added autosome (equivalent to tammar chromosome 4) in an $\mathrm{XY}_{1} \mathrm{Y}_{2}$ system (Toder et al. 1997; Rens et al. 1999). Presumably there was a rearrangement in long-term culture of the venerable PtK2 line. We therefore conclude that SYBL1 is part of the ancient X chromosome that is conserved in all therian mammals and is therefore at least 130 million years old.

The other three PAR2 genes all proved to be autosomal in the wallaby, implying that they were translocated to the human sex chromosomes after the divergence of marsupials and eutherians about 130 Mya. None mapped to tammar chromosome $5 p$, the location of PAR1 genes. Thus the addition of PAR2 was independent of PAR1, which was a part of a large region added to both sex chromosomes 80-130 Mya. The stages in the genesis of the PAR2 region can be deduced from the positions of PAR2 homologs in primate, mouse, cat, and marsupial (summarized in Table 1). HSPRY3 is on the $\mathrm{X}$ in all eutherians, but autosomal in marsupials, so must have been added 80-130 Mya, independently of PAR1. CXYorf1 is on the $\mathrm{X}$ and autosomes in primate and mouse (Gianfrancesco et al. 2001a), but autosomal in the wallaby, so must have been added before the divergence of the marsupial lineage from eutherians $\sim 130$ Mya and most likely added with HSPRY3. IL9R is on the $\mathrm{X}$ in all primates, but autosomal in mouse, cat, and wallaby; it was therefore added to the primate $\mathrm{X}$ later (60-70 Mya). The most likely scenario to explain the current position of these genes in the PAR2 of the human $X$ chromosome (Fig. 2) is therefore that the original therian $\mathrm{X}$ contained SYBL1 at a telomeric site (D'Esposito et al. 1997) and other genes were added in the order HSPRY3, \pm CXYorf1, and IL9R. Addition of HSPRY3 and CXYorf1 was followed by inversion to make HSPRY3 proximal to SYBL1. Similarly, addition of IL9R (the last gene) must have been followed by an inversion with the previously added CXYorf1. Because there are multiple copies of IL9R and CXYorf1 on the autosomes, gene duplication may play a role in the evolution of $I L 9 R$ and CXYorf1.

An alternative that we cannot rule out is that PAR2 genes were originally on the ancestral mammalian $\mathrm{X}$, but were independently transferred from the $\mathrm{X}$ to autosomes. However, this would require independent loss of HSPRY3, CXYorf1, and $I L 9 R$ in eutherian and marsupial, so it is considered unlikely. Mapping of PAR2 genes in monotremes should help to further clarify the origin of PAR2.

Thus the genesis of the PAR2 region has been complex, requiring three independent addition and two inversion events within a tiny region. This contrasts with the extreme stability of the $\mathrm{X}$ chromosome, which is almost invariant in eutherian mammals, and which includes a very large region that has been conserved for more than 170 million years. Remarkably, instability seems to be a special feature of both pseudoautosomal regions. Many genes such as CSF2RA and $I L 3 R A$ within and near to the human PAR1 are autosomal in mouse, and some (e.g., $K A L)$ appear to have been lost completely from the mouse genome (Toder and Graves 1998).

\section{The Cause of PAR Instability}

An explanation for this instability of PAR1 and PAR2 may be provided by the hypothesis that at meiosis unpaired chromosome ends tend to associate with nonhomologous regions with which they may recombine, producing terminal translocations (T. Ashley and J. Graves, unpubl.). This hypothesis is based on the observation that unpaired ends of a heteromorphic bivalent (sex chromosomes or rearranged autosomes) tend to associate nonhomologously (Ashley et al. 1981). This could explain the terminal locations and multiple copies of the transferred Csf2ra and Il3ra in mouse as well as explaining the multiple transfers and inversions in the genesis of the PAR2 region.

\section{METHODS}

\section{Animal Tissue}

Tammar wallaby (M. eugenii ) material was obtained from The Melbourne Royal Zoological Gardens under the La Trobe University Animal Ethics Committee permit number RP96/4/V6. Ear tissue was used to establish cell cultures, and liver was 
used for the isolation of genomic DNA. Cat and lemur material was obtained from cultured fibroblasts.

\section{Isolation of Genomic Clones}

A size-selected $\lambda$ genomic library was previously constructed from tammar wallaby male liver (Delbridge et al. 1997). Briefly, the genomic DNA was partially digested in the 15-20 $\mathrm{kb}$ size range with Sau3A and packaged in $\lambda$ EMBL 3 BamHI arms. The library was titered and plated to a density of 120,000 plaque-forming units (pfu) on four $22 \times 22 \mathrm{~cm}$ Nunc plates. The library plates were lifted twice onto nylon membrane (Hybond-N+, Amersham).

To isolate tammar wallaby genomic IL9R, HSPRY3, SYBL1, and CXYorf1 clones, $>360,000$ recombinant phage were screened with human cDNA probes for each of the genes. Small hybridizing fragments of wallaby genomic clones were subcloned into pBluescript. The plasmid primers T3 and T7 were used to obtain sequence from either end of the cloned fragment with the AmpliCycle Sequencing Kit (Promega) according to the manufacturer's instructions. For $S Y B L 1$, small hybridizing fragments subcloned into the pCR4Blunt-TOPO vector of the TOPO Shotgun Subcloning Kits (Invitrogen) were sequenced with $\mathrm{T} 3$ and $\mathrm{T} 7$ primers.

To isolate cat and lemur genomic IL9R, HSPRY3, and SYBL1, specific primers for human PAR2 genes were selected and used to prepare probes for library screening. These probes were used to screen high-density filters of the entire RPCI-86 Feline Male BAC Library and LBNL-2 Lemur (Lemur catta) (BACPAC Resources). Hybridization of high-density filters were performed following standard protocol reported at http://www.chori.org/bacpac/. Clones used for FISH were confirmed by PCR.

\section{Fluorescence In Situ Hybridization}

Chromosome preparations were obtained from ear fibroblasts of a male tammar wallaby cultured in DME with $10 \%$ of fetal bovine serum. After harvesting, the cell pellet was dropped onto clean wet slides, which were stored at $-20^{\circ} \mathrm{C}$ until use. For in situ hybridization, we followed the protocol detailed in Svartman and Vianna-Morgante (1999), with minor modifications. The probes were labeled with biotin-14-dCTP by nick translation (BioNick; Life Technologies) and, after precipitation with suppressor DNA (tammar wallaby genomic DNA sheared to $500 \mathrm{bp}$ ) in a proportion of 1:250, $200 \mathrm{ng}$ of each probe were applied to the hybridization areas. Hybridization was carried out at $42^{\circ} \mathrm{C}$ overnight (CXYorf1, HSPRY3, and SYBL1) or at $37^{\circ} \mathrm{C}$ for $3 \mathrm{~d}$ (IL9R). Posthybridization washes were performed at $42^{\circ} \mathrm{C}$, one in $50 \%$ formamide/2xSSS and one in $2 \mathrm{xSSC}$, both for $3 \mathrm{~min}$. Immunodetection was performed with polyclonal antibiotin raised in sheep (3:500, Vector), followed by antisheep IgG conjugated with FITC (1:100, Vector). Counterstaining was performed with propidium iodide $(0.6 \mathrm{ng} / \mu \mathrm{L})$ and the preparations were mounted with DAPI $(0.8 \mathrm{ng} / \mu \mathrm{L})$ in Vectashield Mounting Medium (Vector). Double hybridization was performed with digoxigeninlabeled HSPRY3 and biotin-labeled CXYorf1 in the same conditions described above, and for immunodetection we used antibiotin conjugated with FITC and antidigoxigenin conjugated with rhodamine (Oncor). For sequential FISH, after hybridization and analysis of $H S P R Y 3$, the chromosome preparation was left overnight in PBS at $4^{\circ} \mathrm{C}$ and hybridization with tammar wallaby chromosome 3 paint (Toder et al. 1997) was then performed as described above for single sequences. Analyses were performed in a Zeiss Axioplan microscope and images were collected with a liquid charge-coupled device (CCD) camera (Photometrics).

For the cat and lemur, chromosome preparation was obtained following standard protocols. Slides were hybridized in situ basically as described by Lichter et al. (1990), with minor modifications. We used $300 \mathrm{ng}$ of BAC probe in each experi- ment; hybridization was performed at $37^{\circ} \mathrm{C}$ in 2 XSSC, $50 \%$ $(\mathrm{v} / \mathrm{v})$ formamide, $10 \%(\mathrm{v} / \mathrm{v})$ dextran sulfate, $5 \mathrm{mg}$ human Cot1 DNA (Gibco-BRL), and $3 \mathrm{mg}$ sonicated salmon sperm DNA, in a volume of $10 \mathrm{~mL}$. Posthybridization washings were at $0.1 \mathrm{XSSC}$ at $60^{\circ} \mathrm{C}$, three times each. Digital images were obtained using a Leica DMRXA epifluorescence microscope equipped with a cooled CCD camera (Princeton Instruments). DAPI was used to counterstain lemur and feline chromosomes to recognize them on the basis of the DAPI banding pattern. Cy3 and DAPI fluorescence signals, detected with specific filters, were recorded separately as gray-scale images. Pseudocoloring and merging of images were performed using Adobe Photoshop software.

\section{ACKNOWLEDGMENTS}

We thank Iole Barbieri for excellent technical assistance and Dr. Angela M. Vianna-Morgante for the use of laboratory facilities for double painting experiments. This work was supported by a grant from the National Health and Medical Research Foundation of Australia. The financial support of CEGBA (Centro di Eccellenza Geni in campo Biosanitario e Agroalimentare) and MIUR (Ministero Italiano della Istruzione e della Ricerca) is gratefully acknowledged. The financial support of Telethon (Mde) is gratefully acknowledged. M. Svartman was a recipient of a postdoctoral fellowship from Fundação de Amparo à Pesquisa do Estado de São Paulo (FAPESP). F.J. Charchar is supported by a Wellcome Trust Travelling Research Fellowship.

The publication costs of this article were defrayed in part by payment of page charges. This article must therefore be hereby marked "advertisement" in accordance with 18 USC section 1734 solely to indicate this fact.

\section{REFERENCES}

Ashley, T., Moses, M.J., and Solari, A.J. 1981. Fine structure and behaviour of a pericentric inversion in the sand rat, Psammomys obesus. J. Cell Sci. 50: 105-119.

Charchar, F.J., Svartman, M., and Graves, J.A.M. 2000. Assignment of SPROUTY 1 (SPRY1) gene to tammar wallaby chromosome 6 by fluorescence in situ hybridization. Cytogenet. Cell Genet. 90: $240-241$.

Ciccodicola, A., D'Esposito, M., Esposito, T., Gianfrancesco, F., Migliaccio, C., Miano, M.G., Matarazzo, M.R., Vacca, M., Franze, A., Cuccurese, M., et. al. 2000. Differentially regulated and evolved genes in the fully sequenced Xq/Yq pseudoautosomal region. Hum. Mol. Genet. 12: 395-401.

Cooke, H.J., Brown, W.R., and Rappold, G.A. 1985. Hypervariable telomeric sequences from the human sex chromosomes are pseudoautosomal. Nature 317: 687-692.

Delbridge, M.L., Harry, J.L., Toder, R., O’Neill, R.J., Ma, K., Chandley, A.C., and Graves, J.A.M. 1997. A human candidate spermatogenesis gene, $R B M 1$, is conserved and amplified on the marsupial Y chromosome. Nat. Genet. 15: 131-136.

D'Esposito, M., Matarazzo, M.R., Ciccodicola, A., Strazzullo, M., Mazzarella, R., Quaderi, N.A., Fujiwara, H., Ko, M.S., Rowe, L.B., Ricco, A., et al. 1997. Differential expression pattern of XqPAR-linked genes SYBL1 and IL9R correlates with the structure and evolution of the region. Hum. Mol. Genet. 6: 1917-1923.

Freije, D., Helms, C., Watson, M.S., and Donis-Keller, H. 1992. Identification of a second pseudoautosomal region near the Xq and Yq telomeres. Science 258: 1784-1787.

Gianfrancesco, F., Falco, G., Esposito, T., Rocchi, M., and D’Urso, M. 2001a. Characterization of the murine orthologue of a novel human subtelomeric multigene family. Cytogenet. Cell Genet. 94: $98-100$

Gianfrancesco, F., Sanges, R., Esposito, T., Tempesta, S., Rao, E., Rappold, G., Archidiacono, N., Graves, J.A.M., Forabosco, A., and D'Urso, M. 2001b. Differential divergence of three human pseudoautosomal genes and their mouse homologs: Implications for sex chromosome evolution. Genome Res. 11: 2095-2100.

Graves, J.A.M. 1995. The origin and function of the mammalian Y chromosome and Y-borne genes-an evolving understanding. Bioessays 17: 311-320.

Graves, J.A.M., Wakefield, M.J., and Toder, R. 1998. The origin and 


\section{Charchar et al.}

evolution of the pseudoautosomal regions of human sex chromosomes. Hum. Mol. Genet. 7: 1991-1996.

Huber, R., Hansen, R.S., Strazzullo, M., Pengue, G., Mazzarella, R., D’Urso, M., Schlessinger, D., Pilia, G., Gartler, S.M., and D'Esposito, M. 1999. DNA methylation in transcriptional repression of two differentially expressed X-linked genes, GPC3 and SYBL1. Proc. Natl. Acad. Sci. 96: 616-621.

Kermouni, A., Van Roost, E., Arden, K.C., Vermeesch, J.R., Weiss, S., Godelaine, D., Flint, J., Lurquin, C., Szikora, J.P., Higgs, D.R., et al. 1995. The IL-9 receptor gene (IL9R): Genomic structure, chromosomal localization in the pseudoautosomal region of the long arm of the sex chromosomes, and identification of IL9R pseudogenes at 9qter, 10pter, 16pter, and 18pter. Genomics. 29: $371-382$.

Kuhl, H., Rottger, S., Heilbronner, H., Enders, H., and Schempp, W. 2001. Loss of the Y chromosomal PAR2-region in four familial cases of satellited Y chromosomes (Yqs). Chromosome Res. 9: $215-222$.

Kvaloy, K., Galvagni, F., and Brown, W.R. 1994. The sequence organization of the long arm pseudoautosomal region of the human sex chromosomes. Hum. Mol. Genet. 3: 771-778.

Kumar, S. and Hedges, S.B.A. 1998. Molecular timescale for vertebrate evolution. Nature 392: 917-920.

Li, L. and Hamer, D.H. 1995. Recombination and allelic association in the Xq/Yq homology region. Hum. Mol. Genet. 4: 2013-2016.

Lichter, P., Tang Chang, C.J., Call, K., Hermanson, G., Evans, G.A., Housman, D., and Ward, D.C. 1990. High resolution mapping of human chromosomes 11 by in situ hybridization with cosmid clones. Science 247: 64-69.

Matarazzo, M.R., Cuccurese, M., Strazzullo, M., Vacca, M., Curci, A., Giuseppina Miano, M., Cocchia, M., Mercadante, G., Torino, A., et al. 1999. Human and mouse SYBL1 gene structure and expression. Gene 240: 233-238.

Rappold, G.A. 1993. The pseudoautosomal regions of the human sex chromosomes. Hum. Genet. 92: 315-324.

Rens, W., O'Brien, P.C., Yang, F., Graves, J.A.M., and Ferguson-Smith, M.A. 1999. Karyotype relationships between four distantly related marsupials revealed by reciprocal chromosome painting. Chromosome Res. 7: 461-474.

Svartman, M. and Vianna-Morgante, A.M. 1999. Comparative genome analysis in American marsupials through banding and in situ hybridization. Chromosome Res. 7: 267-275.

Toder, R. and Graves, J.A.M. 1998. CSF2RA, ANT3, and STS are autosomal in marsupials: Implications for the origin of the pseudoautosomal region of mammalian sex chromosomes. Mamm. Genome. 9: 373-376.

Toder, R., O'Neill, R.J.W., Wienberg, J., O'Brien, P.C.M., Voullaire, L., and Graves, J.A.M. 1997. Comparative chromosome painting between two marsupials: Origins of an $\mathrm{XX} / \mathrm{XY}_{1} \mathrm{Y}_{2}$ sex chromosome system. Mammal Genome. 8: 418-442.

Vermeesch, J.R., Petit, P., Kermouni, A., Renauld, J.C., Van Den Berghe, H., and Marynen, P. 1997. The IL-9 receptor gene, located in the Xq/Yq pseudoautosomal region, has an autosomal origin, escapes $\mathrm{X}$ inactivation and is expressed from the Y. Hum. Mol. Genet. 6: 1-8.

Vogt, P.H., Affara, N., Davey, P., Hammer, M., Jobling, M.A., Lau, Y.F., Mitchell, M., Schempp, W., Tyler-Smith, C., Williams, G., et al. 1997. Report of the third international workshop on Y chromosome mapping. 1997. Heidelberg, Germany Cytogenet. Cell Genet. 79: 1-20.

Waters, P.D., Duffy, B., Frost, C.J., Delbridge, M.L., and Graves, J.A.M. 2001. The human Y chromosome derives largely from a single autosomal region added to the sex chromosomes 80-130 million years ago. Cytogenet. Cell Genet. 92: 74-79.

Received May 2, 2002; accepted in revised form October 23, 2002.

\section{Genome Research}




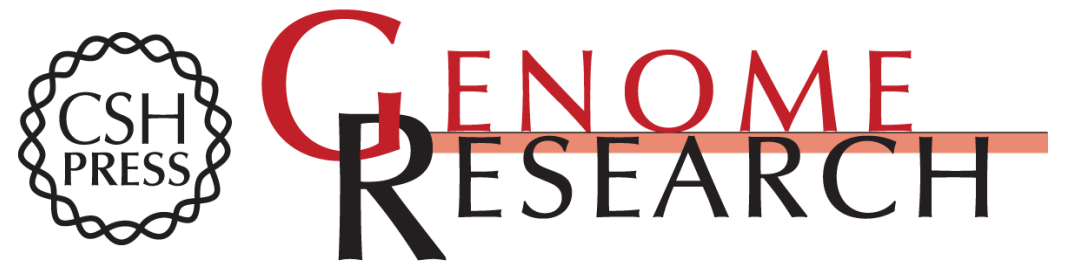

\section{Complex Events in the Evolution of the Human Pseudoautosomal Region 2 (PAR2)}

Fadi J. Charchar, Marta Svartman, Nisrine El-Mogharbel, et al.

Genome Res. 2003 13: 281-286

Access the most recent version at doi:10.1101/gr.390503

References This article cites 25 articles, 5 of which can be accessed free at:

http://genome.cshlp.org/content/13/2/281.full.html\#ref-list-1

License

Email Alerting Receive free email alerts when new articles cite this article - sign up in the box at the Service top right corner of the article or click here.

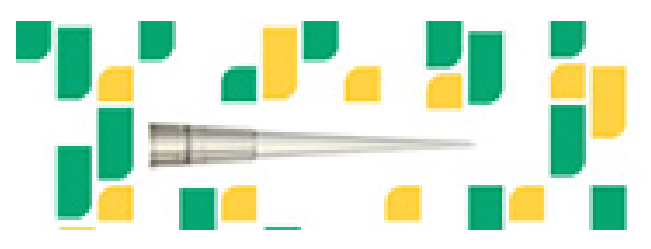

Focused on your science.

To subscribe to Genome Research go to: https://genome.cshlp.org/subscriptions 